Regards sur l'économie allemande

Bulletin économique du CIRAC

$77 \mid 2006$

Varia

\title{
Fiscalité : réforme structurelle et consolidation budgétaire
}

Markus Gabel

\section{OpenEdition}

\section{Journals}

Édition électronique

URL : http://journals.openedition.org/rea/784

DOI : $10.4000 /$ rea.784

ISBN : 978-2-8218-0850-8

ISSN : 1965-0787

Éditeur

CIRAC

Édition imprimée

Date de publication : 1 juillet 2006

Pagination : 5-12

ISSN : 1156-8992

Référence électronique

Markus Gabel, «Fiscalité : réforme structurelle et consolidation budgétaire », Regards sur l'économie allemande [En ligne], 77 | juillet 2006, document 1, mis en ligne le 01 juillet 2008, consulté le 02 mai 2019. URL : http://journals.openedition.org/rea/784; DOI : 10.4000/rea.784 


\title{
Fiscalité : réforme structurelle et consolidation budgétaire
}

\author{
Markus Gabel
}

\begin{abstract}
L'objectif majeur du gouvernement de coalition fédéral est la consolidation des finances publiques à partir de 2007. A cet effet, il a adopté un ensemble de mesures échelonnées dans le temps et qui mélangent habilement approches keynésiennes, cap d'austérité et politique de l'offre. Ainsi, au plan de relance 2006 destiné à stimuler les investissements et la demande intérieure succèdera l'an prochain une politique d'austérité avec augmentations d'impôts (notamment de la TVA) et réduction des dépenses publiques. Le gouvernement fait le pari qu'avec le retour général de la confiance, la croissance retrouvée sera suffisamment robuste pour supporter cette cure d'austérité. En 2008 suivra le volet d'offre du programme au centre duquel se trouve la fiscalité des entreprises, notamment des grandes sociétés, afin d'améliorer l'attractivité du Standort Deutschland.

Ce programme budgétaire et fiscal, qui peut se lire comme une parfaite traduction des sensibilités très opposées de la grande coalition, modifiera profondément la structure de la fiscalité. En volume, il se traduira certes par une hausse des prélèvements fiscaux (mesurés par rapport au PIB), mais il est basé sur un rééquilibrage entre, d'un côté, impôts directs et cotisations sociales (en baisse) et, de l'autre, impôts indirects (en hausse). L'Allemagne retrouve ainsi des structures fiscales plus proches de la moyenne européenne.
\end{abstract}

\section{Héritage fiscal et marge de manœuvre de la grande coalition}

Depuis une quinzaine d'années, le système fiscal allemand enregistre un retard par rapport aux régimes de la plupart des autres pays industrialisés. II tient aux effets d'une économie globalisée où le niveau d'imposition et la mobilité des facteurs entretiennent ipso facto une relation inverse : plus le facteur est mobile (comme le capital), plus il ne supporte qu'une fiscalité réduite. L'Allemagne, championne mondiale des exportations, ne satisfait que très imparfaitement à cette règle ; dans un récent classement de l'efficacité des systèmes fiscaux, elle se situe en queue de peloton. Cette inefficacité remonte à l'immédiat aprèsguerre. Le gouvernement allemand d'alors défendait une baisse radicale des taux d'imposition suite à l'introduction du deutsche mark, mais les autorités alliées, craignant l'insuffisance des recettes et donc des moyens nécessaires à la reconstruction, s'opposaient à cette mesure. Le résultat fut l'instauration d'un système basé sur des taux élevés, notamment sur le capital, conjugués à de nombreuses exceptions, déductions et exonérations auxquelles entreprises et particuliers se sont vite habitués. En 1971 déjà, une commission d'experts se plaignait d'un système inefficace et à peine compréhensible, créant de nombreuses distorsions dans l'allocation des facteurs.

Avec la réunification, cette structure est arrivée à son paroxysme. Afin de favoriser l'investissement dans les nouveaux Länder face au défi exceptionnel de la reconstruction, le gouvernement de l'époque a fait le choix de la subvention fiscale (dérogations, exonérations, etc.) tout en maintenant les taux d'imposition à leur niveau élevé. Cette politique était non seulement à l'opposé de celle des pays voisins (qui commençaient à pratiquer un mix entre baisse des taux
Un système fiscal en décalage par rapport aux pays industrialisés

Niveau d'imposition élevé, mais multiplication des dérogations 
De faibles recettes fiscales compensées par des prélèvements sociaux élevés

d'imposition des entreprises et élargissement de l'assiette), mais a surtout montré sa complète inefficacité (érosion de la base fiscale, baisse de la progressivité fiscale réelle et surtout mauvaise allocation des ressources conduisant à un excès d'offre de logements à l'Est).

Au total, malgré l'augmentation des impôts indirects et l'introduction de la surtaxe de solidarité sur l'impôt sur l'ensemble des revenus et bénéfices (Solidaritätszuschlag), la part des recettes fiscales (sans cotisations sociales) dans le PIB allemand a stagné durant les années 1990, conduisant ainsi inévitablement vers l'autre tendance de cette période, celle de la forte hausse des cotisations sociales, handicapant lourdement l'évolution du marché du travail ainsi que le développement du secteur des services. Cette situation perdure jusqu'à aujourd'hui, car aucun progrès n'a pu être enregistré entre 1998 et 2005 sous la coalition rouge-verte, malgré notamment l'introduction de l'impôt écologique dont les recettes sont destinées partiellement à diminuer le niveau des cotisations retraites, mais dont le volume est resté largement en-dessous des aspirations. Ainsi, en 2004, l'Allemagne détient le record européen en termes de cotisations sociales (42,6\% de l'ensemble des recettes publiques).

Structures fiscales allemandes face à l'Europe depuis 1995

\begin{tabular}{|c|c|c|c|c|c|c|c|c|c|c|c|c|}
\hline \multirow{5}{*}{$\begin{array}{l}\text { Allemagne } \\
\text { EU-25 }{ }^{5)} \\
\text { EU-12 }{ }^{6)}\end{array}$} & \multicolumn{3}{|c|}{ Ratio fiscal global ${ }^{1)}$} & \multicolumn{3}{|c|}{ Impôts directs ${ }^{2)}$} & \multicolumn{3}{|c|}{ Impôts indirects ${ }^{3)}$} & \multicolumn{3}{|c|}{ Cotisations sociales ${ }^{4)}$} \\
\hline & 1995 & 2000 & 2004 & 1995 & 2000 & 2004 & 1995 & 2000 & 2004 & 1995 & 2000 & 2004 \\
\hline & 39,8 & 41,9 & 38,7 & 27,5 & 29,8 & 26,3 & 30,2 & 29,9 & 31,1 & 42,3 & 40,4 & 42,6 \\
\hline & 39,7 & 41,0 & 39,3 & 31,3 & 34,3 & 32,7 & 33,8 & 34,5 & 35,0 & 35,0 & 31,5 & 32,5 \\
\hline & 39,9 & 41,5 & 39,7 & 28,5 & 31,3 & 29,8 & 32,8 & 34,0 & 34,2 & 38,9 & 35,3 & 36,2 \\
\hline
\end{tabular}

Sources : Commission européenne (2005). 1) Total des recettes fiscales (avec cotisations sociales) en pourcentage du PIB. 2) Ensemble des impôts directs en pourcentage des recettes fiscales totales. 3) Ensemble des impôts indirects en pourcentage des recettes fiscales totales. 4) Ensemble des cotisations sociales en pourcentage des recettes fiscales totales. 5) Moyenne pondéré de l'EU-25. 6) Moyenne pondéré de la zone euro.

Entreprises : une charge fiscale cumulée de $39 \%$

La Cour de Karlsruhe, acteur majeur de politique fiscale

Un budget fédéral à la limite de la constitutionnalité
Au milieu des années 1990, le gouvernement Kohl avait certes tenté une réforme, mais son projet s'était heurté à l'obstruction du Bundesrat (la CDU/CSU n'y avait plus la majorité depuis 1991). La réforme n'a pu aboutir qu'en 2000, sous le gouvernement Schröder, conduisant à une baisse du taux de l'impôt sur le revenu (de $53 \%$ à $42 \%$ ) et du taux de l'impôt pour les sociétés (de $45 \%$ à $25 \%$ ). Ce taux de $25 \%$ paraît attractif en termes de concurrence, mais il faut y ajouter la taxe professionnelle et la surtaxe de solidarité (3 points de pourcentage) auxquelles sont soumises les entreprises allemandes. Ainsi, la charge cumulée réelle s'élève à environ $39 \%$, ce qui est non seulement loin de la moyenne européenne de $26 \%$, mais aussi le niveau le plus élevé en Europe. Une nouvelle réforme, notamment du régime fiscal des entreprises, reste plus que jamais à l'ordre du jour.

Durant les années 1990, les gouvernements successifs ont dû faire face à la montée en puissance d'un autre acteur dans la politique fiscale : le Tribunal constitutionnel fédéral. II a rendu plusieurs arrêts très coûteux pour le budget fédéral, comme la suppression de l'impôt sur le patrimoine, l'exonération du minimum vital (Existenzminimum) ou la réduction de la charge fiscale pesant sur les familles. Cette juridicisation explique pourquoi, depuis, les gouvernements allemands agissent avec une prudence accrue et tentent d'anticiper l'interprétation de la Cour de Karslruhe.

Actuellement, la grande coalition n'a qu'une marge de manœuvre financière très réduite, car en sus de l'augmentation des cotisations sociales, les gouvernements successifs avaient largement fait appel à l'endettement pour financer les transferts de l'ouest à l'est. Malgré de nombreux plans d'économies (les premiers remontent à 1993) basés sur une réduction des transferts sociaux, des dépenses liées aux fonctionnaires, de certaines subventions et, surtout, des dépenses militaires, aucun des deux gouvernements n'a réussi une consolidation durable. Le critère de $3 \%$ de déficit du traité de Maastricht n'est plus respecté depuis 2001. Et presque tous les ans, le gouvernement allemand doit déclarer 
une situation de déséquilibre économique global afin de satisfaire, au moins formellement, à l'article 115 de la Loi fondamentale qui exige que l'endettement doit rester inférieur aux dépenses d'investissement.

\section{Gouvernement Merkel : priorité à la consolidation}

Le programme du gouvernement Merkel est formel : l'heure est à la consolidation budgétaire et non à la baisse fiscale généralisée. Bien que le dispositif ne soit pas encore définitif, un premier volet doit être voté par le Bundestag à la fin juin. II concerne notamment la hausse de la TVA de trois points de pourcentage (un tiers des recettes induites est destiné à la réduction du taux de cotisation à l'assurance chômage), ainsi que du taux supérieur de l'impôt sur le revenu (il passera de 42 à $45 \%$ ) et de l'impôt sur les assurances. Ces dispositions entreront en vigueur le $1^{\mathrm{er}}$ janvier 2007. Ces hausses sont accompagnées de plusieurs mesures restrictives concernant les épargnants, les parents, les enseignants (suppression de la déductibilité des dépenses liées au bureau à domicile), ainsi que la déductibilité des frais de déplacement professionnels.

En termes de recettes fiscales, la hausse de la TVA (et de l'impôt sur les assurances, de 3 points de pourcentage chacun) est de loin la mesure phare du gouvernement, car la hausse de I'IR sera limitée aux revenus supérieurs à $250000 €$ annuels pour les célibataires (le double pour un couple) et ne frappera pas les revenus d'activités commerciales et industrielles. Ces mesures constituent la plus forte hausse fiscale de l'après-guerre ; selon les estimations, le volume des recettes devrait atteindre 538 milliards $€$ d'ici 2010 , soit près d'un quart de plus qu'aujourd'hui. Quant à la réforme fiscale des entreprises, elle est prévue pour le $1^{\text {er }}$ janvier 2008 seulement. Le gouvernement prévoit en effet une réforme en profondeur, comprenant non seulement une baisse des taux et un élargissement de l'assiette, mais également l'introduction du principe de neutralité de la fiscalité par rapport à la raison sociale des entreprises, ainsi que par rapport à la détermination du bénéfice des sociétés.

Le gouvernement bâtit son action sur une stratégie audacieuse. Sachant que la cure d'austérité qu'il prévoit pour 2007 risque de nuire à la croissance, il multiplie actuellement les efforts pour restaurer la confiance et faciliter l'investissement des entreprises (notamment par des conditions d'amortissement plus favorables), afin de profiter encore plus de l'accélération de la croissance mondiale. Le bon moral des chefs d'entreprise et le regain de confiance des ménages, mais aussi la très bonne tenue des recettes fiscales (au plus haut depuis six ans, avec 463 milliards $€$ attendus pour 2006), confortent pour l'instant le gouvernement dans la stratégie choisie. Grâce à ces recettes plus élevées que prévu, il peut même espérer respecter les critères de Maastricht dès 2006.

La première étape de la réforme fiscale qui se dessine ainsi en Allemagne a un double caractère : d'un côté, elle comporte une augmentation de la charge fiscale, de l'autre, elle a une implication structurelle forte. Si l'augmentation du taux plafond de l'IR (fruit d'un marchandage interne entre les deux parties de la coalition) brouille le message et nuit quelque peu à l'attractivité du pays, la hausse de la TVA à $19 \%$ rapproche la structure fiscale allemande de la moyenne européenne - à condition cependant que le gouvernement réussisse dans sa volonté de réduire le niveau des cotisations sociales qui atteignent un niveau record. Le taux actuel de la TVA (16\%) se situe au bas de la moyenne européenne et permettait facilement de justifier la résistance allemande à des demandes de baisse ciblée pour certains secteurs, par exemple la restauration ou le bâtiment. Cette position pourra bientôt appartenir au passé, car avec un taux de désormais $19 \%$, on peut s'attendre à ce que les représentants professionnels allemands formulent des revendications comparables.

Par ailleurs, la hausse prévue de la TVA rejoint un débat plus large concernant la structure de la fiscalité au sein de l'UE. Ce débat s'est ravivé avec l'entrée
Hausse de la TVA en 2007, réforme de la fiscalité des sociétés en 2008

Au préalable : soutenir la confiance des acteurs

La hausse de la TVA rapproche la structure des prélèvements de la moyenne européenne

Une fiscalité plus attractive pour les entreprises en Europe de l'Est 
La fiscalité basée sur la consommation est moins sensible à l'évasion

Hausse du taux plafond de I'IR : une mesure purement symbolique

des 10 nouveaux Etats membres dans I'UE en 2004. Leur structure fiscale se distingue très nettement de celle des anciens, surtout des plus grands : en effet, à l'est de l'Allemagne, les facteurs revenu et bénéfices sont faiblement imposés en comparaison, et les recettes fiscales reposent davantage sur la consommation. En termes de localisation, la fiscalité tchèque, slovaque ou hongroise a ainsi un effet très direct sur les préférences des entrepreneurs allemands.

S'il est accompagné d'une révision de la fiscalité pesant sur les entreprises, le choix du gouvernement berlinois est astucieux à double titre. D'abord, en termes d'efficacité, car l'imposition de l'utilisation du revenu au lieu de sa génération encourage et facilite la formation du capital et renforce les incitations en faveur du travail et des investissements. Un tel système est dès lors plus favorable à la croissance. Ensuite, il rend l'évasion fiscale plus difficile, car les possibilités d'évasion sont plus réduites pour la consommation qu'elles ne le sont pour le capital. Les systèmes fiscaux basés sur la consommation sont ainsi plus aptes à résister à la concurrence fiscale internationale.

La hausse du taux plafond de l'IR est certainement la mesure la plus ambiguë du gouvernement. Dans le fil de la reforme fiscale SPD/Verts, ce taux venait juste de tomber de 45 à $42 \%$ en 2005. Mais cette réforme était le résultat d'un compromis entre les deux chambres parlementaires ; le gouvernement Schröder avait baissé ce taux plus que ne le souhaitait le SPD, afin de s'assurer le soutien du Bundesrat, dominé par l'opposition d'alors (CDU/CSU). Dans la négociation des compromis au sein de l'actuelle grande coalition, le SPD aura finalement obtenu gain de cause dans sa volonté de faire participer davantage les hauts revenus à l'effort d'assainissement budgétaire - malgré le fait que ce groupe y participe déjà de manière disproportionnée. Cela dit, la mesure est plutôt de nature symbolique car les recettes générées seront très faibles.

$0,34 \%$ des contribuables ont apporté 17,2\% des recettes fiscales en 2005

\begin{tabular}{|ccc|}
\hline Contribuables avec un revenu annuel de & Nombre & Part dans les recettes fiscales totales \\
$\mathbf{0}-\mathbf{5 0} \mathbf{0 0 0} €$ & 23,8 millions $(82,64 \%)$ & $33,4 \%$ \\
$\mathbf{5 0} \mathbf{0 0 0}-\mathbf{1 2 5} \mathbf{0 0 0} €$ & 4,5 millions $(15,62 \%)$ & $38,2 \%$ \\
$\mathbf{1 2 5} \mathbf{0 0 0}-\mathbf{2 5 0} \mathbf{0 0 0} €$ & 0,4 million $(1,39 \%)$ & $11,2 \%$ \\
$>\mathbf{2 5 0} \mathbf{0 0 0} €$ & $\mathbf{0 , 1}$ million (0,34\%) & $\mathbf{1 7 , 2} \%$ \\
\hline
\end{tabular}

Source : Destatis.

Une mesure par ailleurs contraire à la Constitution..

... et comportant un grave risque budgétaire
Indépendamment de ce revirement comme de l'inefficacité de la mesure, cet « impôt des riches » (Reichensteuer) est contestable pour des raisons constitutionnelles. II instaure en effet un traitement fiscal déséquilibré des revenus qui est doublement contraire au principe fondamental d'égalité devant la loi (art. 3 de la Loi fondamentale) puisqu'il distingue entre revenus privés (imposés) et revenus d'activités commerciales et industrielles (exemptés) et que, au sein de cette dernière catégorie, seuls les revenus des professions libérales seront imposés. Le gouvernement défend son choix par le fait que si l'impôt était étendu à l'ensemble des revenus professionnels, une partie des sociétés de personnes (entre 5 et $10 \%$ ) supporterait elle aussi cette hausse, ce qui est peu compatible avec la création de conditions d'investissement attractives. Ce faisant, il cherche aussi à prévenir un changement de régime ultérieur, car la « grande » réforme fiscale des entreprises, prévue pour 2008, vise justement la baisse de la charge fiscale pour ce type de sociétés. Par ailleurs, la question de la constitutionnalité se pose également au sujet des mesures restrictives concernant la déductibilité des dépenses personnelles liées à l'activité salariale (bureau à domicile, déplacements professionnels) qui est contraire au principe fiscal du traitement net des revenus (Nettoprinzip: revenus moins les dépenses nécessaires pour les obtenir).

Le cas de la Reichensteuer montre parfaitement les limites du projet de la grande coalition, basé sur des approches fiscales très opposées. Non seulement celui-ci signale un respect douteux de la Constitution, mais comporte sur- 
tout un grave risque budgétaire en cas de recours auprès du Tribunal constitutionnel fédéral, car le gouvernement devra restituer les recettes perçues en cas d'arrêt négatif. Les juges de Karlsruhe ont été saisis récemment d'un cas similaire : la réforme fiscale de 2000 avait introduit un traitement inégal pour les revenus commerciaux et industriels, d'un côté (taux plafond de $45 \%$ ), et, de l'autre, les revenus salariés et ceux des professions libérales (53\%). Considérant ce traitement comme contraire à la Loi fondamentale, la Cour fédérale des finances avait renvoyé l'affaire devant les juges de Karlsruhe. Une décision est attendue pour cette année.

Bien que la grande coalition présente sa politique de hausses fiscales (les plus fortes de l'après-guerre, rappelons-le) comme indispensable, d'autres choix auraient été possibles, comme le montre l'expérience des années 1980. A l'époque, dans une situation budgétaire tout aussi tendue, le gouvernement avait eu le courage de procéder à des économies plus importantes qu'aujourd'hui tout en soutenant l'offre par des baisses d'impôt. Un tel scénario n'était pourtant pas hors de portée aujourd'hui : il suffit de penser à l'accord KochSteinbrück de 2003 prônant une réduction drastique des subventions (elle aurait rapporté au budget fédéral 15,8 milliards $€$ sur 3 ans), ainsi qu'à l'accord Schröder-Merkel-Stoiber conclu lors du Sommet pour l'emploi de 2005 et proposant de ramener le taux de l'impôt sur les sociétés de $25 \%$ à $19 \%$.

\section{Une réforme fiscale des entreprises en demi-teinte}

Afin de profiter de la plus grande efficacité des systèmes fiscaux basés sur la consommation, la grande coalition doit compléter la hausse de la TVA par une baisse des impôts directs. Compte tenu de la baisse de l'IR achevée en 2005, l'action du gouvernement se concentre sur la fiscalité des entreprises. Le contrat de coalition mentionne clairement cet objectif. Ce document énonce également plusieurs principes qui devraient orienter la réforme, notamment le fait que la réforme concernerait l'ensemble du secteur des entreprises afin d'améliorer l'attractivité de l'Allemagne et qu'elle devrait restaurer la neutralité de l'impôt par rapport au statut et au type de financement des sociétés.

L'imposition des entreprises en Europe (en \%) ${ }^{1)}$

\begin{tabular}{|cl|cc|cc|cc|}
\hline Allemagne & $38,6^{2)}$ & France & 33,3 & Portugal & 27,5 & Pologne & 19,0 \\
Italie & $37,3^{2}$ & Grande-Bretagne & 30,0 & Finlande & 26,0 & Slovaquie & 19,0 \\
Espagne & 35,0 & Pay-Bas & 29,0 & Autriche & 25,0 & Hongrie & 17,5 \\
Belgique & 34,0 & Suède & 28,0 & Rep. tchèque & 24,0 & Lituanie & 12,5 \\
\hline
\end{tabular}

Sources : Commission européenne (2005). 1) Taux nominal ajusté d'imposition sur le revenu des sociétés en 2006. La moyenne arithmétique pour la zone euro se situe à $29,7 \%$ et à $25,9 \%$ pour l'EU-25. 2) Avec les 3 points de pourcentage de l'impôt de solidarité.

Pour mettre en œuvre la réforme, le gouvernement étudie deux modèles : l'imposition synthétique ou l'imposition duale (voir REA 65/04). Le modèle synthétique (comprehensif tax), défendu notamment par la Stiftung Marktwirtschaft, englobe l'ensemble des revenus (travail, capitaux, revenus commerciaux ou industriels, etc.). Dans le contexte de la mondialisation, un tel système exerce une pression à la baisse des taux, ce qui mène à un système de flat tax à un niveau suffisamment bas pour qu'il ne chasse pas hors des frontières les facteurs les plus mobiles. La progressivité d'un tel système ne se fait que par l'abattement de base. Le déroulement de la campagne électorale a cependant montré que le système d'une flat tax (défendu alors par Paul Kirchhof) se heurte à une résistance politique trop élevée. Bien qu'il exerce des effets indéniablement bénéfiques sur l'offre et ainsi sur la croissance, ce qui générerait d'autres recettes fiscales, un tel système risque cependant d'être insuffisant en termes de recettes, notamment si l'Allemagne continue dans la voie du cofinancement des systèmes sociaux par des recettes fiscales.

Le Conseil des Sages et d'autres experts encore favorisent ainsi le concept d'une imposition duale, tel qu'il a été mis en place dans les années 1990 dans
Une hausse de la fiscalité n'était pas la seule possibilité

Objectif : neutralité de l'impôt par rapport au statut des sociétés
Rejet du système de la flat tax, pourtant favorable à la croissance

Le Conseil des Sages prône un système dual à la mode scandinave 
les pays scandinaves. Ici, l'idée de base est de moduler le taux d'imposition selon le degré de mobilité des bases d'imposition. Un tel système se traduit en pratique par la distinction très nette entre revenu de capitaux (avec une imposition relativement faible et à taux unique) et du travail (avec des taux plus élevés et à l'intérieur d'un système progressif). Du point de vue de l'efficacité économique, l'imposition différenciée de ce système est problématique car, pour profiter des taux plus bas sur le capital, elle incite à des comportements indésirables, comme le développement de la fausse indépendance (Scheinselbständigkeit). Reste le problème de la constitutionnalité d'une telle différenciation. La grande coalition a ici une carte particulière à jouer, car l'interprétation des juges de Karlsruhe serait certainement plus favorable si un tel système était voté avec une large majorité à la fois du Bundestag et du Bundesrat.

\section{L'actuel système d'imposition des entreprises dominé par l'inégalité}

Le code fiscal allemand ne traite pas toutes les entreprises de la même manière. Les sociétés de personnes sont régies selon le principe de transparence. Ainsi, le résultat de l'entreprise est attribué aux propriétaires qui doivent les imposer dans leur déclaration personnelle de revenus. Le fait que les bénéfices soient thésaurisés ou distribués n'a aucune influence sur le taux d'imposition. L'entreprise pour sa part n'est soumise qu'à la taxe professionnelle (Gewerbesteuer), dont l'assiette n'est pas diminuée par d'éventuelles bonifications aux propriétaires. Cependant, la taxe professionnelle payée par l'entreprise est déductible du revenu imposable du ou des propriétaires. Ces derniers bénéficient également de la possibilité de compenser une perte provenant d'une participation dans une société de personnes avec leurs autres revenus. Le niveau d'imposition des sociétés de personnes dépend alors du taux de l'IR auquel est soumis le propriétaire. Pour 80 à $85 \%$ de ces sociétés, la charge moyenne est inférieure à $30 \%$.

Pour une société à capitaux (Kapitalgesellschaft), régie selon le principe de la séparation (Trennungsprinzip), la situation est très différente, car elle représente un sujet fiscal autonome (selbständiges Steuersubjekt) et est ainsi soumise à l'impôt sur les sociétés, actuellement au taux de $25 \%$. La société est également soumise à la taxe professionnelle qui, contrairement aux sociétés de personnes, ne peut pas être imputée sur l'impôt sur le revenu des propriétaires de la société et représente ainsi une charge réelle supplémentaire pour l'entreprise. Cependant, la charge de la taxe professionnelle est souvent inférieure à celle des sociétés de personnes car des bonifications éventuelles versées aux propriétaires réduisent le bénéfice et ainsi l'assiette de la taxe professionnelle. Cependant, les propriétaires doivent déclarer les dividendes et intérêts reçus de l'entreprise et les imputer dans leur déclaration des revenus personnels. Les dividendes ne sont imposables que pour moitié (Halbeinkünfteverfahren). La société peut compenser des bénéfices par des pertes afin de réduire la charge fiscale, cette possibilité - contrairement aux sociétés de personnes n'existe pas pour les propriétaires. Globalement, la charge fiscale moyenne des sociétés à capitaux se situe à un peu plus de 38 \% (25\% pour l'IS et environ $13 \%$ pour la taxe professionnelle).

Concilier compétitivité fiscale et consolidation budgétaire

Réduire la charge fiscale des sociétés à capitaux à moins de $30 \%$
Plus de sept mois après les déclarations d'intention du contrat de coalition, la fenêtre d'opportunité pour la réforme fiscale n'est plus aussi largement ouverte, car le pragmatisme fiscal a pris le dessus sur la grande réforme structurelle. Ainsi, le rétablissement de la compétitivité internationale doit se faire sans menace sur la consolidation budgétaire en cours. Deux facteurs expliquent cette évolution. D'abord, le nouveau ministre des Finances partage globalement l'opinion de son prédécesseur, selon laquelle les entreprises allemandes supportent certes des taux nominaux élevés, mais profitent d'une assiette relativement faible. Ensuite, les experts du ministère estiment le coût des deux options de la Stiftung Marktwirtschaft et du Conseil des Sages à 20, voire 30 milliards $€$ de pertes nettes de recettes fiscales - des sommes incompatibles avec la situation des finances publiques. Certes, les conséquences d'une grande réforme sont difficilement chiffrables, car les réactions des entreprises sont entachées d'une grande incertitude. Mais après la dernière réforme fiscale des entreprises de 2000, l'impôt sur les sociétés s'est totalement effondré durant deux ans.

Malgré ces incertitudes et face à une certaine cacophonie fiscale au sein de la coalition, la Chancelière a recadré la réforme : elle abandonne l'objectif de neutralité des recettes et vise désormais un allègement réel de la charge fiscale des entreprises (entre 6 et 10 milliards $€$ ). Quant à l'arbitrage entre les sociétés de personnes et les sociétés à capitaux, ce sont ces dernières qui recueillent les faveurs du gouvernement. L'objectif est de réduire leur charge fiscale totale à un niveau inférieur à $30 \%$. Avec une taxe professionnelle qui pèse en moyenne 14 à $15 \%$, le taux d'imposition des sociétés devrait ainsi tomber de $25 \%$ à quelque $15 \%$ (12,5\% sont également en discussion pour leur effet de signal car représentant une baisse nominale de $50 \%$ ), donc nettement plus bas qu'envisagé lors du Sommet pour l'emploi de 2005. Afin de compenser partiellement les 10 milliards $€$ de pertes (chaque point de pourcentage du taux de l'IS 
coûte environ 800 millions $€$ au fisc), la baisse du taux se doublera d'un élargissement de l'assiette (réduction et suppression d'exonérations et de subventions et renforcement de la lutte contre l'évasion et la fraude fiscale).

Les grands perdants de cette orientation de la réforme fiscale sont les PME, donc le Mittelstand. Le gouvernement souhaite concentrer l'effort d'allègement sur les très grandes sociétés de personnes. Non seulement il prévoit de réduire le taux d'imposition sur les bénéfices réinvestis dans l'entreprise (en cas d'utilisation par le propriétaire, ils resteront soumis au taux maximum de $42 \%$ de I'IR, puis de $45 \%$ à partir de 2007), il favorise également un modèle qui donnerait un droit d'option aux entreprises. Ainsi, les sociétés de personnes auraient le choix de se faire imposer comme une société à capitaux, donc avec le nouveau taux de l'IS plus la taxe professionnelle. Un tel modèle est avantageux pour environ 20000 grandes sociétés de personnes. Enfin, il instaurerait ainsi la neutralité de l'impôt face au statut de l'entreprise.

Si ces mesures ne révolutionnent pas la fiscalité, elles sont néanmoins un pas en avant. Un rôle fondamental dans le développement de cette réforme a été joué par l'unité des grandes fédérations, traditionnellement divisées car intégrant, avec l'artisanat et les grandes entreprises industrielles, des intérêts trop divergents. Mais cette fois-ci, le gouvernement ne pouvait pas compter sur leur division. Au contraire, dans une lettre commune adressée début mai à la Chancelière par les représentants de l'industrie, du commerce et de l'artisanat (BDI, DIHK et ZDH), elles ont trouvé une position commune, certes moins ambitieuse que les options de la Stiftung Marktwirtschaft et du Conseil des Sages, mais déterminée par les réalités. Le résultat de cette unité est frappant : la lettre expose point par point les mesures envisagées finalement par le gouvernement.

Ce consensus est renforcé par le fait que plus récemment, les fédérations des collectivités territoriales ont-elles aussi signalé leur volonté d'arriver à un compromis. Leur cheval de bataille est surtout la taxe professionnelle qui revient aux communes et qui produit actuellement des recettes record. En termes d'attractivité du site, elle devrait être abolie. En 1997, une partie de cet impôt, celle pesant sur les capitaux, a déjà été supprimée. Mais comme en France, la réforme de la taxe professionnelle est délicate, parce qu'elle nécessiterait en parallèle une réforme des finances communales. Depuis la réforme de 1997, l'existence de cet impôt est inscrite dans la Loi fondamentale (Art. 28), de sorte qu'aujourd'hui toute tentative de changement se heurterait à la difficulté de trouver la majorité nécessaire.

Les propositions visant une réforme ou suppression de la taxe professionnelle sont nombreuses, mais souvent trop ambitieuses. Le modèle de la Stiftung Marktwirtschaft est le plus radical. II propose de remplacer la taxe professionnelle (qui représente aujourd'hui le pilier essentiel du financement des communes) par un système basé sur 4 ressources : une taxe foncière réformée, un nouvel impôt sur les bénéfices des entreprises (à taux modulable par la commune), un impôt citoyen (Bürgersteuer), également nouveau, et une participation des communes aux recettes de l'impôt sur le revenu (actuellement réparties entre niveau fédéral et les Länder). Mais ce modèle ne trouve pas vraiment de soutien au sein du gouvernement, qui reste pour l'instant divisé entre l'Union $\mathrm{CDU} / \mathrm{CSU}$ favorable à une réforme, voire à une suppression de la taxe professionnelle, et des sociaux-démocrates favorables à une baisse du taux parallèlement à un élargissement de l'assiette. Cette dernière position est également partagée par les représentants des communes qui favorisent une baisse des taux, accompagnée d'un élargissement de la notion de bénéfices (qui engloberait également les intérêts, les baux et les contrats de leasing), ainsi que l'extension de la taxe professionnelle aux professions libérales.

Le risque est ainsi réel qu'un mauvais standard (la taxe professionnelle) finisse par perdurer et même par s'amplifier. Une telle orientation est tout sauf idéale pour les communes, car la taxe professionnelle est répartie de manière très
Un modèle qui favorise les grandes entreprises

Le projet est le fruit de l'unité des fédérations professionnelles

Les communes plaident pour le maintien de la taxe professionnelle

Comment réformer la taxe professionnelle?

Après la réforme de l'IS, elle sera la principale taxe frappant les entreprises 
inégale sur le territoire, fluctue fortement avec le cycle conjoncturel et favorise les grandes villes. Mais le plus grand problème de cette taxe est qu'elle enfreint le principe d'égalité devant l'impôt. D'un côté, les sociétés à capitaux doivent la supporter entièrement, ce qui pèse sur leurs charges, de l'autre, elle ne pèse que formellement sur les sociétés de personnes car si elles la payent, elles ne la supportent pas réellement (cette taxe peut être déduite de l'impôt sur le revenu des propriétaires de la société). La taxe professionnelle est ainsi devenue un instrument très peu transparent dans la péréquation entre communes, Länder et Bund. Elle devrait dès lors être au centre de la réforme de la fiscalité des entreprises. Elle le sera au plus tard après l'entrée en vigueur en 2008 de la réforme de l'impôt sur les sociétés, car alors cette taxe sera le principal impôt pesant sur les entreprises. Déjà pour 2006, dans un contexte de forte hausse des recettes fiscales versées par les entreprises (on attend plus de 94 milliards $€$ ), les recettes de la taxe professionnelle, également en forte hausse, représentent près de $40 \%$ dans l'ensemble de la fiscalité d'entreprise - un record jamais atteint.

ECONOMIQUEMENT COMME FISCALEMENT, la politique de la grande coalition se place plus dans la continuité des réformes engagées par la coalition SPD/Verts qu'elle ne représente une rupture avec elle. Mais à la différence du précédent, l'actuel gouvernement a su restaurer la confiance des entreprises et des ménages. Cette confiance retrouvée, il est maintenant de la plus grande importance de ne pas la décevoir avec la réforme fiscale des entreprises. Cette dernière risque cependant d'être une réforme inachevée tant que ne sera pas affrontée la "réforme des réformes »: celle du fédéralisme financier. Sans décision sur la réorganisation des relations financières entre l'échelon fédéral, les Länder et les communes ainsi que sur leurs ressources financières respectives, toute réforme de la taxe professionnelle est bloquée. Les décisions concernant le fédéralisme financier sont attendues pour cet automne - probablement trop tard pour la réforme de la fiscalité des entreprises.

Malgré cette expectative, le programme fiscal du gouvernement Merkel : rééquilibrage entre imposition directe et indirecte et neutralité de l'impôt face à la raison sociale de l'entreprise, reste suffisamment important pour être qualifié de réforme structurelle et non pas seulement de variation du taux d'imposition. Dans l'histoire fiscale allemande, on ne compte d'ailleurs que deux réformes structurelles dignes de ce nom : celle de 1967 qui a introduit un système moderne d'imposition des plus-values et celle de 1998 qui a introduit le principe de l'imposition à moitié (Halbeinkünfteverfahren) pour les dividendes. Quoi qu'il en soit, le véritable problème de l'Allemagne n'est pas son système fiscal, mais l'inadéquation entre le niveau des recettes et celui des dépenses. Le choix de la consolidation, combinée à certaines coupes dans les dépenses publiques, est ainsi certainement la bonne voie à prendre.

\section{Indications bibliographiques :}

BouRGEOIs I., "Quels choix fiscaux pour l'Allemagne ? » et « Réforme fiscale allemande : la lente construction des équilibres d'Helmut Kohl à Gerhard Schröder ", Regards sur l'économie allemande, $\mathrm{n}^{\circ \mathrm{s}} 65$, mars 2004, et 54, décembre 2001

CDU, CSU, SPD, Koalitionsvertrag, Gemeinsam für Deutschland - mit Mut und Menschlichkeit, 11 novembre 2005

Commission EUROPÉENNE, Structures of the taxation systems in the European Union: 1995-2004, 2006

DeUTSCHE BANK RESEARCH, Staatliche Finanzierungssysteme im Wettbewerb, 8-12-2005

GABEL M., "Le lent assainissement des finances publiques allemandes ", Regards sur l'économie allemande, $\mathrm{n} \% 3$, mai 2000

SACHVERSTÄNDIGENRAT ZUR BEGUTACHTUNG DER GESAMTWIRTSCHFATLICHEN ENTWICKLUNG, Staatsfinanzen konsolidieren - Steuersystem reformieren. Jahresgutachten 2003-2004, et Die Chance nutzen - Reformen mutig voranbringen, Jahresgutachten 2005/06. 